\title{
6
}

\section{Fluorescence Spectroscopy as a Screening Tool and Continuous Monitor for Urban Water Quality}

\author{
Chris Smart, Brad Simpson and Andy Joyce
}

The fluorescence spectrum of water samples can be readily measured to identify the presence of many natural and anthropogenic organic compounds. Water samples from storm drains, creeks and seeps along the Thames River in London, Ontario, contained gasoline, undifferentiated PAHs, sewage and fluorescent dyes. Some sites showed consistent spectra, others were highly variable. Time series of samples and continuous records show that fluorescence varies in response to runoff and human activities. The spatial and temporal variability of water quality indicates that analysis of a limited number of discrete, point water samples is unlikely to characterise real contamination patterns. Temporal and spatial variability require greater attention.

\subsection{Introduction}

Surface and groundwater contamination by a wide range of anthropogenic compounds presents a monitoring problem as it requires compositional, temporal and spatial resolution. Resources have primarily been directed towards resolving the compounds present in a limited number of water samples. If contamination is not detected in samples it is taken to indicate low hazard. However, sampling protocols based on regular sampling at a designated site (typically monthly at a gauging station or monitoring well) will not necessarily reveal transient or spatially restricted contamination (Table 6.1). The occasional positive sample may even be dismissed as erroneous.

Smart, C., B. Simpson and A. Joyce. 2004. "Fluorescence Spectroscopy as a Screening Tool and Continuous Monitor for Urban Water Quality." Journal of Water Management Modeling R220-06. doi: 10.14796/JWMM.R220-06.

(C) CHI 2004 www.chijournal.org ISSN: 2292-6062 (Formerly in Innovative Modeling of

Urban Water Systems. ISBN: 0-9683681-9-0) 
Table 6.1 A summary of the sampling protocol indicated by spatial and temporal character of contamination.

\begin{tabular}{|l|l|l|l|}
\hline \multirow{2}{*}{ Contaminant sampling modes } & \multicolumn{2}{c|}{ Temporal character } \\
\cline { 3 - 4 } \multicolumn{2}{c|}{} & \multicolumn{1}{|c|}{ Chronic } & \multicolumn{1}{c|}{ Acute } \\
\hline \multirow{3}{*}{$\begin{array}{l}\text { Spatial } \\
\text { Character }\end{array}$} & $\begin{array}{l}\text { Extensive } \\
\text { Contamination }\end{array}$ & $\begin{array}{l}\text { Discrete Point } \\
\text { Sarnpling }\end{array}$ & $\begin{array}{l}\text { Continuous Point } \\
\text { Sampling }\end{array}$ \\
\cline { 2 - 5 } & $\begin{array}{l}\text { Point } \\
\text { Contamination }\end{array}$ & $\begin{array}{l}\text { Discrete Distributed } \\
\text { Sampling }\end{array}$ & $\begin{array}{l}\text { Continuous Distributed } \\
\text { Sampling }\end{array}$ \\
\hline
\end{tabular}

The emphasis placed on composition means that we have little idea of the origin of point contamination. Unfortunately, improved spatial resolution demands a large number of spatially distributed samples, precluding thorough analysis. A solution to this problem is to develop screening technologies permitting rapid triage of samples, so that only critical samples are forwarded for analysis. Such samples may be selected as occurring during hydrological or "monitoring" events likely to compromise water quality, but should also be used to demonstrate that inter-event periods are of low risk.

Transient contamination requires very high frequency or continuous sampling. There are very few field-capable continuous analytical systems yielding compound specific analysis, especially where the composition is not necessarily known in advance. However, a number of easily measured surrogate variables provide broad indications of the water quality, permitting tracking of changes in water quality. Electrical conductivity, turbidity, dissolved oxygen, water stage and temperature are examples. A number of these variables can be continuously measured at sufficiently low cost that it is possible to install a sparse distributed monitoring network for little more than the cost of a few complete analyses. Spatial resolution of a sparse network is relatively poor, but adaptive redeployment of monitors permits spatial focussing.

Polycyclic organic contaminants are a major concern, but are not detected by standard sensors. However, many of these compounds are fluorescent allowing their detection at very low concentrations by fluorescence spectroscopy (Kershaw and Fetzer, 1995, Baker 2002). The purpose of this chapter is to summarise our exploration of fluorescence spectroscopy as a screening and continuous monitoring tool, with a focus on the Thames River and Medway Creeks in London, Ontario. 


\subsection{Fluorescence Spectroscopy}

A large number of cyclic organic compounds are noted for their fluorescence; their ability to absorb light in particular wavelengths, and to re-emit light at a lower wavelength (e.g. Lakowicz, 1983). The excitation (absorbed) and emission (emitted) wavelengths are compound specific, although not unique, and the intensity of emission is proportional to concentration. Some fluorescent dyes are readily detected at concentrations of a few ppt. It is possible to characterise fluorescence characteristics by position in Excitation-Emission space. Figure 6.1 shows the location of a number of compounds in the ultraviolet to visible range of the electromagnetic spectrum. Unfortunately, the "footprint" of many compounds occupy relatively wide areas so that they overlap and mask one another. Most surface waters contain fluorescent natural organic matter (Frimmel 1998). These undifferentiated humic and fulvic materials occupy a particularly wide area of the spectrum and may mask a number of contaminants (Roch 1997). Furthermore, absorption of light can significantly suppress the spectrum (Mobed et al. 1996).

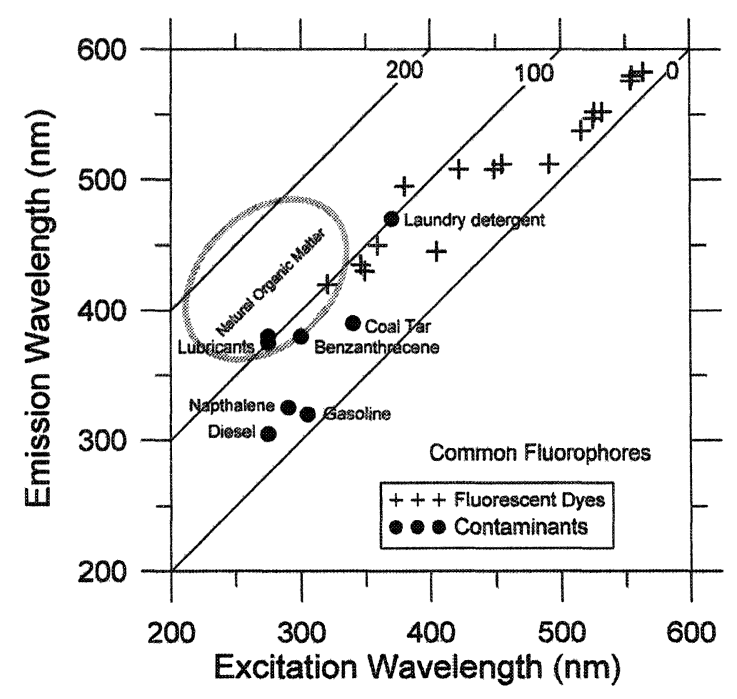

Figure 6.1 The excitation-emission diagram allows the position of many common fluorophores to be identified. The numbered diagonal lines indicate the path of a "synchronous scan", at the indicated offset $(\Delta \lambda)$ between excitation and emission wavelengths. 
Given this mix of promise and constraint, it is important to design the analytical strategy carefully so that the best information is obtained. In the field, the wavelengths of excitation and emission are typically fixed by use of restricted spectrum light sources and optical filters. In the laboratory, it is possible to scan a range of wavelengths using monochromators. A single scan cuts across excitation-emission space producing a single fluorescence spectrum. The most efficient mode of analysis is a 45 degree path obtained using "synchronous scanning" where excitation and emission wavelengths are slewed at equal rates with a fixed offset $(\Delta \lambda)$. The result is a spectrum of fluorescence intensity against emission wavelength, with peaks indicating fluorescent compounds. (e.g. Figures 6.2-6.5). We typically use $\Delta \lambda=20 \mathrm{~nm}$ to detect dyes and light hydrocarbon fuels, and $\Delta \lambda=90 \mathrm{~nm}$ to detect natural organic mater, raw sewage, lubricants and degraded fuels. Coal-tar derivatives are best detected at $\Delta \lambda=50 \mathrm{~nm}$.

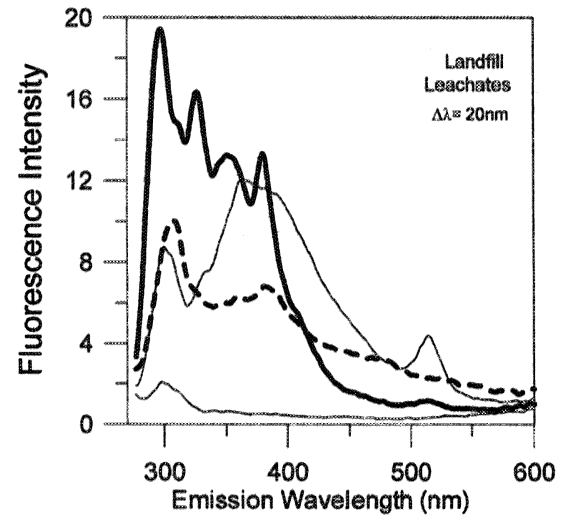

Figure 6.2 A range of leachates from landfill and brownfield sites. Multiple peaks below $400 \mathrm{~nm}$ arise from fuels and solvents. The $512 \mathrm{~nm}$ peak is from uranine dye found in antifreeze. The undifferentiated peak around $350-400 \mathrm{~nm}$ is from degraded organic matter; it suppresses the spectrum at lower wavelengths. The bottom spectrum is for deionised water.

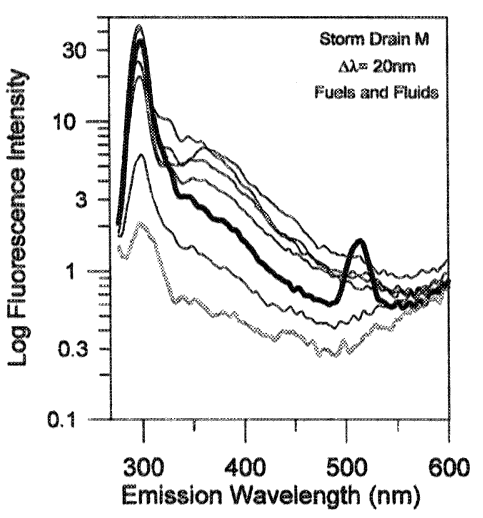

Figure 6.3 A series of samples from a single storm drain showing acute presence of fuels, and uranine dye. Detailed analysis suggested that at some points diesel was present. 


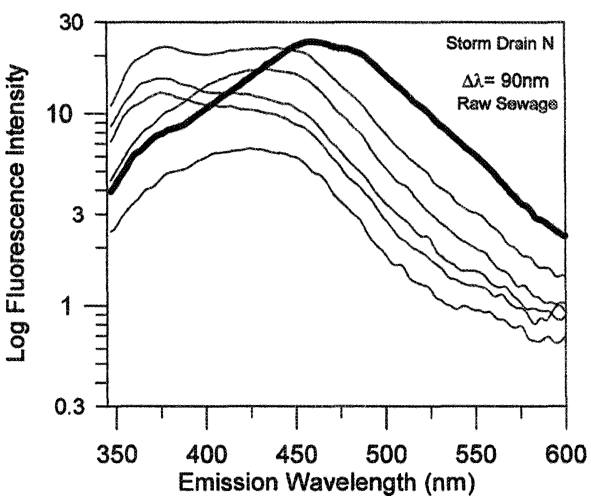

Figure 6.4 A series of samples from a single storm drain showing very high dissolved organic matter and in one sample optical brighteners (thick line) implying a sanitary drain connection.

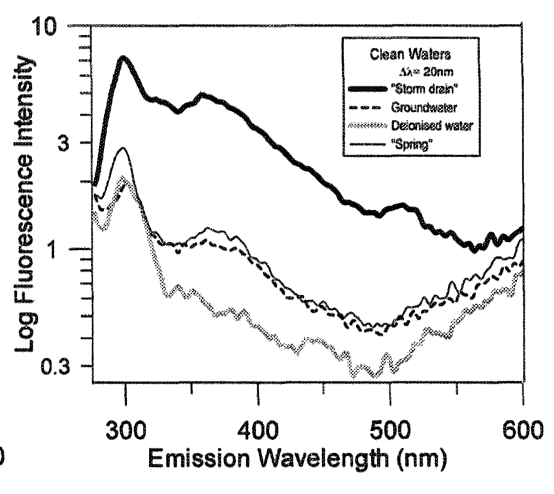

Figure 6.5 Groundwater has a much lower fluorescence than surface waters.An apparent spring entering the Thames river has a "groundwater signature" at $\Delta \lambda=20 \mathrm{~nm}$ but differentiation at $\Delta \lambda=90 \mathrm{~nm}$ suggests that it is mains water.

\subsection{Sampling and Analysis}

Spatial sampling was undertaken along the south fork of the Thames River through London, Ontario, focussing on bankside seepage, storm drains and tributaries. The river bank is characterised by brown field and industrial sites. River water samples were dominated by ambient waters, so that inputs were highly diluted; even river water samples collected adjacent to known coal tar seeps showed little detectable product. All water samples described below were from storm drains or seeps, collected below the water surface in glass scintillation phials leaving zero head space. Replicate samples were collected over periods of a few days, with activated charcoal detectors left in place to scavenge any transient contamination in the intervening time (Smart and Simpson 2002). Samples were analysed in quartz cuvettes on a Photon Technology International QM1 spectrofluorometer with a xenon arc source and $3 \mathrm{~nm}$ slit settings. Activated charcoal-filtered tap water was used as a blank and the mercury vapour emission lines from a fluorescent light fixture as a check on wavelength. A Uranine dye standard was used as a fluorescence intensity standard. Synchronous scans were run at $\Delta \lambda=20$ and $90 \mathrm{~nm}$, with occasional samples run at multiple $\Delta \lambda$ to build a data base for excitation- 
emission plots. Data were compiled in Excel and three dimensional renditions developed in Surfer. Fluorescence data are generally expressed as fluorescence intensity, a dimensionless count of photon flux dependent on machine settings.

Medway Creek, a tributary to the Thames River in London, Ontario, was the focus of continuous monitoring. This suburban-rural creek drains a 200 $\mathrm{km}^{2}$ catchment, and is considered somewhat degraded, although the nature and origin of contamination is unknown. A number of fluorescence time series were compiled using fluorescence spectra of water samples and Turner Designs Model 10 series filter fluorometers in continuous flow mode (Smart et al.1998). These units were set up with a heated intake line from a pump and used to monitor for blue $(\Delta \lambda \approx 90 \mathrm{~nm})$ and green $(\Delta \lambda \approx 20 \mathrm{~nm})$ fluorescence. In tandem, Levelogger $($ water level temperature, electrical conductivity and dissolved oxygen transducer recorders were installed at the stream bed. Although the fluorometers were calibrated using uranine and optical brightener standards, the fluorescence data are expressed in arbitrary units with linear correction for fouling of the optical path.

\subsection{Results}

A number of brownfield and landfill sites along the river bank drain through seeps and drains into the Thames River. Leachate seeps from a conventional Landfill, capped about forty years ago shows a "volatile fuels"peak at $300 \mathrm{~nm}$, a generic organic peak at $400 \mathrm{~nm}$ and a uranine dye peak at $512 \mathrm{~nm}$ (Figure 6.2). Uranine dye is a very powerful fluorophore (Käss 1998), commonly used to tint antifreeze. This composition is common in leachates from older, aerated landfills. The more peaked spectrum contains many more poorly differentiated volatile organic compounds, fuels, lubricants and solvents. A receiving pool for these materials shows a lower, less differentiated spectrum as the VOCs are lost to evaporation.

Storm drains and creeks feeding into the Thames river showed great variability in space and time. Many responded to runoff by conveying road runoff as might be expected. Figure 6.3 shows spectra from a series of replicate samples collected over a two week period. Under low flow gasoline was detected (Pharr et al. 1992). Under high flow diesel appeared, accompanied by a uranine dye peak. The implication is that the area drained contained not only leaking underground storage tanks, but also accumulations of fuel and fluids mobilised by runoff. 
A nearby storm drain showed a curious range of organics at $\Delta \lambda=90$ (Figure 6.4). Some samples exhibited a $375 \mathrm{~nm}$ peak, most a $430 \mathrm{~nm}$ peak and one a $470 \mathrm{~nm}$ peak. The former two peaks arise from different grades of organic matter. The latter is almost certainly an optical brightener present in many laundry detergents (Poiger et al., 1996). This style of variation is typical of sanitary sewers and sewage discharge (Reynolds and Ahmad, 1997), reflecting the wide range and variability of materials present. The implication is that this storm drain is conveying sanitary sewage directly to the river, presumably indicating an accidental crosslinkage.

Storm drains were generally highly loaded with organic compounds compared to groundwater samples (Figure 6.5), although even groundwater springs showed traces of uranine. One inflow of tens of litres per second appeared unusually clean, having more in common with treated mains water than any other type. In the absence of any hydrogeological expedient, our inference is that this was a major leak from a water main.

A common feature from all the sites was a high level of variability. Some of this appeared to be runoff related, but some sites showed ad hoc variation. Replicate sampling and analyses suggest that this variation is real. A time series of over 180 daily samples from Medway Creek showed very little serial correlation (Smart and Karunaratne 2000). Figure 6.6 shows a brief sequence of winter continuous flow data. Urban runoff in winter is complicated by warm water discharges, road salt-induced melt, natural thaws and rain-on-snow. Stage data are compromised by ice cover. Elevated EC shows saline runoff. For much of the time blue and green fluorescence co-vary, indicating variation in concentration of a fairly consistent composition, loosely referred to as dissolved organic matter. However, there are occasional strong excursions in green fluorescence, largely driven by uranine dye. This is taken to indicate road runoff, but may be from a number of sources. The main inference drawn from Figure 6.6 is that water quality and runoff conditions vary with remarkably high frequency. No representative single water sample could have been collected through the period of observation.

\subsection{Conclusions}

The relative simplicity of sampling and fluorescence analysis allow a large throughput of samples to be screened for very low unit cost. Although the capital cost of a conventional scanning spectrofluorophotometer is few tens of thousands of dollars, small, portable emission scanning spectrometers are now 
available for a few thousand dollars. In situ fluorescence sensors are also available for a few thousand dollars, obviating the need for pumping and heating. Although the capital costs are typical of sensors and laboratory analyses, the lack of preparation, ease of analysis and low maintenance permit a very high information yield.

A wide range of interpretations were possible from fluorescence spectra indicating surface water, groundwater, leachate, leaking underground storage tanks and sanitary sewer discharge. The high sample frequency and density revealed variability of composition that might not have been inferred from a limited number of samples. Spatial and temporal representativity of water analyses demand greater attention, if environmental conditions are to be adequately described.

Continuous fluorometric analysis, in tandem with other surrogate variables suggests that runoff processes contain transient signals that may allow identification of runoff source and associated risk of contamination. The cost of continuous monitoring is relatively high, unless sensors can be simply added to existing monitoring equipment such as stage recorders. Alternatively, integrative samplers such as activated charcoal can be used to accumulate fluorescent contaminants over a period of days (Smart and Simpson 2002). Distributed monitoring exploiting drainage network structure will be necessary to develop interpretations of the runoff patterns observed.

\section{References}

Baker, A., 2002. Fluorescence properties of some farm wastes: implications for water quality monitoring. Water Research, 36 189-195.

Frimmel, F.H., 1998. Characterisation of natural organic matter as major constituents in aquatic systems. Journal of Contaminant Hydrology 35 201-216.

Käss, W., 1998. Tracing technique in Geohydrology, Balkema, Rotterdam.

Kershaw, J.R., and Fetzer, J.C., 1995. The room temperature fluorescence analysis of polycyclic aromatic compounds in petroleum and related materials. Polycyclic Aromatic Compounds 7 p253-268

Lakowicz, 1983. Principles of Fluorescence Spectroscopy. Plenum New York.

Mobed, J.J., Hemmindsden, S.L., Autry, J.L., and McGowan, L.B., 1996. Fluorescence characterisation of IHSS humic substances: total luminescence spectra with absorbance correction. Environmental Science and Technology, 30 3061-3065.

Pharr, D.Y., 1992. Fingerprinting petroleum contamination using synchronous scanning fluorescence spectroscopy. Groundwater $30 \mathrm{p} \mathrm{484-} 489$.

Poiger, T., Field, J.A., Field, T.M., and Giger, W., 1996. Occurrence of flourescent 
whitening agents in sewage and river water determined by solid-phase extraction and high performance liquid chromatography. Environmental Science and Technology $302220-2226$.

Reynolds, D.M., and Ahmad, S.R., 1997. Rapid and direct determination of wastewater BOD values using a fluorescence technique. Water Resources, 31 2102-2018.

Roch, Ch., 1997. The evaluation of total luminescence data with chemometrical methods: a tool for environmental monitoring. Analytica Chemica Acta 356 6174.

Smart, C.C., and K.C. Karunaratne, 2002. Characteristation of fluorescence background in dye tracing Environmental Geology 42, p492-498.

Smart, C.C., and B. Simpson, 2002 Detection of fluorescent compounds in the environment using granular activated charcoal detectors Environmental Geology 42 , p538-545.

Smart, C.C., Zabo, L., Alexander, C.A., and Worthington, S.R.H., 1998. Some advances in fluorometric techniques for groundwater tracing. Environmental Monitoring and Assessment. 53 (2) 305-320. 